\title{
$\phi$ Meson Measurements at RHIC with the PHENIX Detector
}

\author{
Murad Sarsour $^{1, \star}$ on behalf of the PHENIX Collaboration \\ ${ }^{1}$ Georgia State University, Atlanta, Georgia 30303, USA
}

\begin{abstract}
The measurement of $\phi$ mesons provides key information on the phase of the hot and dense medium created in the relativistic heavy ion collisions. It has a relatively small hadronic interaction cross section and is sensitive to the increase of strangeness (strangeness enhancement), a phenomenon associated with soft particles in bulk matter. Measurements in the dilepton channels are especially interesting since leptons interact only electromagnetically, thus carrying the information from their production phase directly to the detector. Measurements in different nucleus-nucleus collisions allow us to perform a systematic study of the nuclear medium effects on $\phi$ meson production. The PHENIX detector provides the capabilities to measure the $\phi$ meson production in a wide range of transverse momentum and rapidity to study these effects.

In this proceeding, we present measurements of the $\phi$ mesons in a variety of collision systems at $\sqrt{s_{N N}}=200 \mathrm{GeV}$. In case of small systems, the data are compared with AMPT calculations to study the various cold nuclear medium effects involved in $\phi$ meson production.
\end{abstract}

\section{Introduction}

Ever since RHIC announced the discovery of the hot and dense state of strongly interacting matter called Quark-Gluon Plasma (QGP) [1-3] the main objective has been to quantify its properties. This is accomplished by looking at as many observables as possible, including the nuclear modification of $\phi$ meson production in the QGP environment. $\phi$ meson is an excellent probe for studying QGP in heavy ion collisions because it is sensitive to several aspects of the collision, including modifications of strangeness production in bulk matter. Owing to its small inelastic cross section for interaction with nonstrange hadrons, the $\phi$ meson is less affected by late hadronic rescattering and may reflect the initial conditions of the evolution of the system. Being a nearly pure strange antistrange $(s \bar{s})$ state, the $\phi$ meson puts additional constraints on models of quark recombination in the QGP. However to gauge the QGP related modifications, we need to know the $\phi$ meson production in $p+p$ collisions as a baseline. There are additional effects from the nuclear medium itself, cold nuclear matter effects $(\mathrm{CNM})$, and they are accessed by studying $\phi$ meson production in small system collisions (e.g. $d+\mathrm{Au}$ ).

The lepton decay channel is of particular interest because of the absence of strong interactions between muons and the surrounding hot hadronic matter. At forward rapidity, it allows us to study the rapidity dependence of $\phi$ meson production and especially in asymmetric heavy-ion collisions provides the means for accessing different mixtures of initial and final state effects.

\footnotetext{
^e-mail: msar@gsu.edu
} 
In $p+p$ collisions, the $\phi$ meson production is important to study because it may have similar production mechanisms to other onia like $J / \psi$ and $\Upsilon$. In addition, it provides a tool to study effects that scale with mass (e.g. collective effects) since it is the heaviest easily accessible meson made of light quarks.

\section{Experimental Setup}

The PHENIX detector [4] has a high rate capability utilizing a fast DAQ and specialized triggers, high granularity detectors, and good mass resolution and particle ID. Detection of $e^{ \pm}$utilizes the finely grained electromagnetic calorimeter (EMCal), the drift chamber and ring imaging Cherenkov detector, while the $\mu^{ \pm}$are detected by the forward spectrometers consisting of Muon ID and Muon Tracker. The very forward beam-beam counters (BBC) are used to determine the collision vertex position and time, the beam luminosity and form a minimum bias trigger.

The PHENIX collaboration collected data from a variety of collisions systems provided by the Relativistic Heavy Ion Collider (RHIC) at Brookhaven National Laboratory (BNL). Results from some of these data sets are presented in this proceeding.

\section{Results}

PHENIX has measured the production of $\phi$ meson in $p+p$ collisions at $\sqrt{s}=200 \mathrm{GeV}$ over a wide range of $p_{T}$ and rapidity $[5,6]$, which is a very important resource for validating the phenomenological models of strangeness production. In addition, these measurements provide baseline for studying cold and hot nuclear modifications.

To gain insight into nuclear medium effects and particle production mechanisms in $A+B$ collisions, the ratio of the $\phi$ meson yields in $A+B$ collisions to $p+p$ collisions scaled by the number of nucleonnucleon collisions in the $A+B$ system, $N_{\text {coll }}$ [7], is calculated as:

$$
R_{A B}=\frac{d^{2} N_{A B} / d y d p_{T}}{N_{\text {coll }} \times d^{2} N_{p p} / d y d p_{T}},
$$

where $d^{2} N_{A B} / d y d p_{T}$ is the per-event yield of particle production in heavy ion collisions and $d^{2} N_{p p} / d y d p_{T}$ is the per-event yield of the same process in $p+p$ collisions. The $p+p$ invariant yield used in the $A+B$ calculation for the $l^{+} l^{-}$decay channel is the $p+p$ differential cross section [5] divided by the $p+p$ total cross section, $42.2 \mathrm{mb}$.

Measurements of $\phi$ meson $R_{A B}$ at midrapidity in heavy ion collisions show that $\phi$ meson exhibits a different suppression pattern compared to lighter mesons $\left(\pi^{0}\right.$ and $\eta$ ) and baryons (protons and antiprotons) [8]. For all centralities, $\phi$ meson is less suppressed than $\pi^{0}$ and $\eta$ in the intermediate $p_{T}$ range (2-5 GeV/c), whereas $\phi, \pi^{0}$ and $\eta$ show similar suppression at higher $p_{T}$. However, $\phi$ meson $R_{A B}$ at forward and backward rapidities [9] show that $\phi$ meson yields in most central $d+$ Au collisions are suppressed at low- $p_{T}(<2 \mathrm{GeV} / \mathrm{c})$ in the $d$-going direction and strongly enhanced in Au-going direction in the intermediate $p_{T}$ range $(2-3 \mathrm{GeV} / \mathrm{c})$. This modification decreases as we move towards peripheral collisions. No modification of $\phi$ meson production is observed in the most peripheral $d+\mathrm{Au}$ collisions. $\phi$ meson $R_{d \mathrm{Au}}$ as a function of $p_{T}$ was measured for different centralities and a nuclear modification very similar to that of the heavy flavor muons is observed [10]. The $\phi$ meson enhancement in the Au-going direction and the suppression in the $d$-going direction are consistent with what is observed by ALICE in $p+\mathrm{Pb}$ collisions at $\sqrt{s_{N N}}=5.02 \mathrm{TeV}$ in $-4.46<y<-2.96$ and $2.03<y<3.53$ [11].

The nuclear-modification factor is also studied in $\mathrm{Cu}+\mathrm{Au}$ collisions, $R_{C u A u}$, at $\sqrt{s_{N N}}=200 \mathrm{GeV}$ to evaluate the effects of hot and cold nuclear matter on $\phi$ meson production. Although statistically 
limited, we observed a dependence of $R_{C u A u}$ on both centrality and rapidity with a similar trend to that observed in $d+$ Au collisions [9].

PHENIX measured the differential cross section of $\phi$ meson at forward and backward rapidities in $p+p$ collisions at $\sqrt{s}=510$ $\mathrm{GeV}$ [12]. The integrated cross section over the measured $p_{T}$ range, $2.79 \pm 0.20$ (stat) \pm 0.17 (syst) \pm 0.34 (norm) $\times 10^{-2} \mathrm{mb}$, from this measurement and those from PHENIX at $\sqrt{s}=200 \mathrm{GeV}[5]$ and LHC at $\sqrt{s}=2.76$ and $7.0 \mathrm{TeV}[11,13-15]$ are used to study the energy dependence of $\phi$ meson production at forward rapidity, $1.2<y<2.2$, in $p+p$ collisions, as shown in Fig. 1. Comparisons to PYTHIA6 [16], PYTHIA8 [17], PHOJET [18], AMPT [19], EPOS3 [20] and EPOS-LHC [21] model predictions show that these models exhibit similar energy dependence trend to that of the data. Further studies with EPOS3 model show that the addition of the hydrodynamic evolution of the system induces an enhancement of $\phi$ meson production

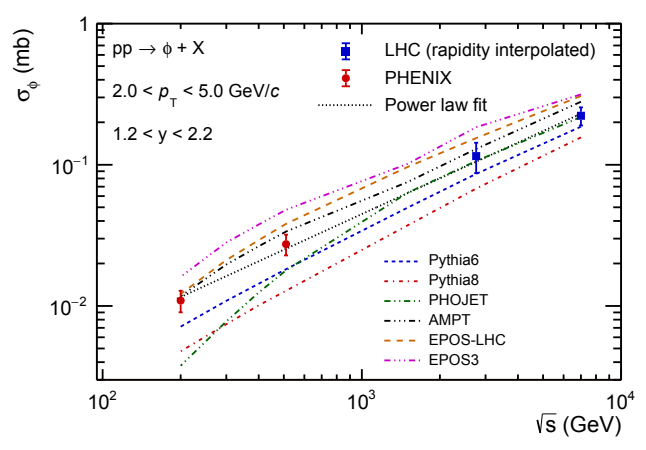

Figure 1. $\phi$ meson production cross section in $1.2<y<$ 2.2 and $2<p_{T}<5 \mathrm{GeV} / \mathrm{c}$ in $p+p$ collisions versus the center-of-mass energy, $\sqrt{s}$, compared to different model predictions. at the LHC energies at intermediate $p_{T}$, which hints at collective effects, however, no effect is observed at RHIC energies [12].

PHENIX collected data from ${ }^{3} \mathrm{He}+\mathrm{Au}, p+\mathrm{Au}$ and $p+\mathrm{Al}$ collisions in 2014 and 2015. In addition to $d+$ Au data set collected in 2008 [8], these data allow to carry out a systematic study of the various cold nuclear matter effects included in models like AMPT and EPOS. The left panel of Fig. 2 shows
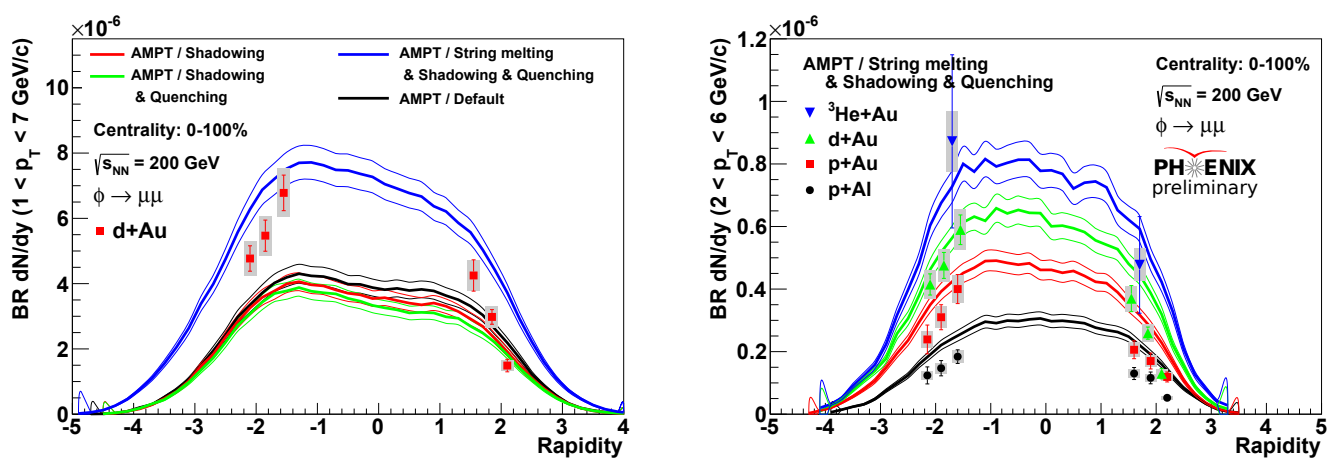

Figure 2. Left: $\phi$ meson invarient yield compared with AMPT model predictions with different combinations of nuclear medium effects. Right: $\phi$ meson invarient yields from ${ }^{3} \mathrm{He}+\mathrm{Au}, d+\mathrm{Au}, p+\mathrm{Au}$ and $p+\mathrm{Al}$ collisions compared with AMPT model predictions that include string melting, shadowing and quenching [19].

AMPT model predictions with different combination of effects included against $\phi$ meson invariant yield in $d+\mathrm{Au}$ collisions. The right panel shows the AMPT model predictions, with the string melting, shadowing and quenching turned on, compared to the data. 


\section{Summary}

The PHENIX collaboration measured $\phi$ meson production, over a wide $p_{T}$ range in the forward and backward rapidities in a variety of collision systems to study cold and hot nuclear matter effects. In $d+$ Au collisions, we observed an enhancement (suppression) of $\phi$ meson production in the backward (forward) rapidity region in most central collisions. Similar behavior was previously observed for inclusive charged hadrons and open heavy flavor muons which may suggest similar cold nuclear matter effects.

The PHENIX collaboration also measured $\phi$ meson production in $p+p$ collisions at $\sqrt{s}=510$ $\mathrm{GeV}$ at backward and forward rapidities, $\sigma_{\phi}=2.79 \pm 0.20$ (stat) \pm 0.17 (syst) \pm 0.34 (norm) $\times 10^{-2} \mathrm{mb}$. Combined with previous PHENIX and LHC measurements, this measurement allowed studying the energy dependence of $\phi$ meson production in $p+p$ collisions over a wide energy range. Comparisons with EPOS3 model showed a hint of collective effects in $p+p$ at LHC energies and no effect at RHIC energies.

New data sets from $p+\mathrm{Au}$ and $p+\mathrm{Al}$ collisions collected in 2015 allow $\phi$ measurement at backward and forward rapidities in less complicated $p+\mathrm{Au}$ and $p+\mathrm{Al}$ systems. The wealth of small system data sets $\left({ }^{3} \mathrm{He}+\mathrm{Au}, d+\mathrm{Au}, p+\mathrm{Au}\right.$ and $\left.p+\mathrm{Al}\right)$ along with the introduction of the forward vertex detector in 2015 data sets will allow studying the different CNM effects using models like AMPT and EPOS.

\section{References}

[1] K. Adcox et al. (PHENIX Collaboration), Nucl. Phys. A 757, 184 (2005).

[2] J. Adams et al. (STAR Collaboration), Nucl. Phys. A 757, 102 (2005).

[3] R. Nouicer, Eur. Phys. J. Plus 131, 70 (2016).

[4] K. Adcox et al. (PHENIX Collaboration), Nucl. Instrum. Methods Phys. Res. A 499, 469 (2003).

[5] A. Adare et al. (PHENIX Collaboration), Phys. Rev. D 90, 052002 (2014).

[6] A. Adare et al. (PHENIX Collaboration), Phys. Rev. D 83, 052004 (2011).

[7] A. Adare et al. (PHENIX Collaboration), Phys. Rev. C 87, 034904 (2013).

[8] A. Adare et al. (PHENIX Collaboration), Phys. Rev. C 83, 024909 (2011).

[9] A. Adare et al. (PHENIX Collaboration), Phys. Rev. C 92, 044909 (2015).

[10] A. Adare et al. (PHENIX Collaboration), Phys. Rev. Lett. 112, 252301 (2014).

[11] J. Adam et al. (ALICE Collaboration), Phys. Lett. B 768, 203 (2017).

[12] A. Adare et al. (PHENIX Collaboration), arXiv:1710.01656v1 (2017).

[13] B. Abelev et al. (ALICE Collaboration), Phys. Lett. B 710, 557 (2012).

[14] B. Abelev et al. (ALICE Collaboration), Eur. Phys. J. C 72, 2183 (2012).

[15] R. Aaij et al. (LHCb Collaboration), Phys. Lett. B 703, 267 (2011).

[16] T. Sjostrand, S. Mrenna, and P. Z. Skands, J. High Energy Phys. 5, 26 (2006).

[17] T. Sjostrand, S. Ask, J. R. Christiansen, R. Corke, N. Desai, P. Ilten, S. Mrenna, S. Prestel, C. O. Rasmussen, and P. Z. Skands, Comput. Phys. Commun. 191, 159 (2015).

[18] F. W. Bopp, R. Engel, and J. Ranft, hep-ph/9803437 (1998).

[19] Z.-W. Lin, C. M. Ko, B.-A. Li, B. Zhang, and S. Pal, Phys. Rev. C 72, 064901 (2005).

[20] K. Werner, B. Guiot, Iu. Karpenko, and T. Pierog, Phys. Rev. C 89, 064903 (2014).

[21] T. Pierog, Iu. Karpenko, J. M. Katzy, E. Yatsenko, and K. Werner, Phys. Rev. C 92, 034906 (2015). 